\title{
Entropía en la periurbanización: desigualdad en el acceso a las infraestructuras de transportes en Tonalá, México
}

\author{
Entropia na periurbanização: desigualdade no acesso às infraestruturas de \\ transportes em Tonalá, México
}
Entropy in peri-urbanization: inequality in access to transport infrastructures in Tonalá, Mexico

Yefer Asprilla Lara ${ }^{[0]}$, Mario Guadalupe González Pérez ${ }^{[b]}$, Fernando García de Quevedo ${ }^{[d]}$

\author{
[a] Universidad Distrital Francisco José de Caldas, Facultad del Medio Ambiente y Recursos Naturales, Bogotá, Colombia \\ [b] Universidad de Guadalajara, Centro Universitario de Tonalá, Tonalá, México \\ ${ }^{[c]}$ Instituto Tecnológico de Monterrey, Campus Guadalajara, Guadalajara, México
}

\section{Resumen}

La movilidad en las franjas periurbanas multicausalmente ha venido experimentando crecimientos alométricos, manifiestos en infraestructuras para sistemas de transporte primordialmente motorizados, las cuales se han caracterizado por evidentes transformaciones y configuraciones morfológicas que confluyen con la generación de entropía en la ciudad-sistema. Aunque esta no es exclusiva de las periferias; es aquí donde se intensifican deficiencias e insuficiencias para unos, y mejoras recalcitrantes para otros. Este trabajo revisa las condiciones físicas y de acceso a las infraestructuras para la movilidad en el sureste del municipio de Tonalá, Jalisco en México, partiendo del enfoque de los sistemas, hemos realizado un ejercicio cuasi-etnográfico auxiliado por registros fotográficos e imágenes satelitales. Los resultados sugieren que el proceso de planificación urbano-territorial en materia de transporte es inequitativo en el acceso a las infraestructuras y no está representando la fuerza neguentrópica necesaria para regular la termodinámica relacionada con el ejercicio de vivir la ciudad o en otros términos, se ha carecido de una concepción sistémica en el proceso de planificación, lo que ha originado costos y tiempos excedentes en la movilidad periurbana.

Palabras clave: Entropía. Infraestructura de transporte. Inequidad.

\section{Resumo}

A mobilidade nas faixas periurbanas tem experimentado um crescimento multifacetado alométrico, manifestado em infraestruturas para sistemas de transporte motorizados, principalmente. Essas infraestruturas se caracterizaram por transformações óbvias e configurações morfológicas, que concorrem para a geração de entropia no sistema urbano. Embora isso não seja exclusivo das periferias, este é o lugar onde as deficiências e inadequações para alguns são intensificados, e melhorias mostram-se recalcitrantes para outros. Este artigo

YAL es MSc, ingeniero civil, especialista en Infraestructura Vial y Transporte, magíster en Ingeniería Civil, estudiante doctorado en Movilidad, Urbana, Transporte y Territorio, Universidad de Guadalajara, profesor asociado de la Universidad Distrital Francisco José de Caldas, e-mail: yasprillal@udistrital.edu.co

MGGP es PhD, ingeniero civil, magíster en Ingeniería Civil, doctor en Ciudad, Territorio y Sustentabilidad, profesor titular de la Universidad de Guadalajara, e-mail: inge_united@hotmail.com

FGQ es MSc ingeniero civil, magister in science in Civil Enginereeng, estudiante de doctorado en Movilidad, Urbana, Transporte y Territorio, Universidad de Guadalajara. Profesor del Instituto Tecnológico de Monterrey, e-mail: fdoquevedo@itesm.mx 
analisa as condições físicas e o acesso às infraestruturas de mobilidade no sudeste do município de Tonalá, Jalisco, México. A partir da abordagem de sistemas, realizamos um exercício quase etnográfico, auxiliado por registros fotográficos e imagens de satélite. Os resultados sugerem que o processo de planejamento urbano-territorial é marcado pelo acesso desigual à infraestrutura e não representa a força neguentrópica necessária para regular a termodinâmica relacionada ao exercício de viver a cidade. Ou seja: tem havido falta de concepção sistêmica no processo de planejamento, o que tem originado custos e tempo excessivos na mobilidade periurbana.

Palavras-chave: Entropia. Infraestrutura de transportes. Desigualdade.

\section{Abstract}

Mobility in the peri-urban strips has been experiencing multifaceted allometric growth, manifested in infrastructures for primarily motorized transport systems, which are characterized by obvious transformations and morphological configurations that converge with the entropy in the city system. Although this is not exclusive of peripheries, this is where deficiencies and inadequacies for some are intensified and recalcitrant improvements for others. This paper reviews the physical conditions and access to mobility infrastructures in the southeast of the municipality of Tonalá, Jalisco-México. From a system approach, we performed a quasi-ethnographic exercise aided by photographic records and satellite images. The results suggest that the urban-territorial planning process is inequitable and does not represent the negentropic force necessary to regulate the thermodynamics related to the exercise of living the city. In other words, there has been a lack of systemic conception in the planning process, originating excessive costs and time in the peri-urban mobility.

Keywords: Entropy. Transport infrastructure. Inequality.

\section{Introducción}

Las ciudades latinoamericanas han seguido por causalidad el modelo de modernidad instaurado desde el siglo pasado en las ciudades norteamericanas, el cual ha influenciado el estilo de vida y la cultura, generalmente con una dependencia importante del vehículo particular. De acuerdo con la evidencia empírica, la configuración de estas formas urbanas detona en dispersión, segregación, desconexión y exclusión (Hiernaux \& Lindon, 2004). En efecto, hay coincidencia en torno al supuesto que otorga, a los dueños del capital, el encargo de la planificación urbana en las ciudades. Desde la infraestructura vial, hidrosanitaria e inmobiliaria, hasta las de carácter recreativo, pasando por las recientemente denominadas infraestructuras sostenibles. En materia habitacional, estos actores han fungido como creadores de vivienda, pero no de ciudad (Barranco \& González, 2016).

En las megalópolis de los países en desarrollo de la región latinoamericana, donde las infraestructuras para la movilidad urbana son deficientes, el urbanismo suele percibirse como "astillado, fraccionado o resquebrajado"(Lorrain, 2005), y en la periurbanización dicha concepción resulta ser más nítida, pues la acción gubernamental resulta insuficiente y en ocasiones nula. Las condiciones aquí (afirmamos), complican el diario vivir, en tanto se ven restringidas las oportunidades de acceso a las necesidades diversas.

En este contexto, si partimos por conveniencia de una visión organísmica del territorio y la ciudad, el marco conceptual de la teoría de los sistemas nos permite proponer que los sistemas territorio y ciudad experimentan entropía. Este concepto implica una medida del escenario morfológico caótico en la ciudad-sistema. Principalmente, en cuanto a su organización estructural y las emanaciones de esa organización. Sí, efectivamente, una ciudad entrópica tiende hacia la desorganización urbana, donde las acciones efectuadas para resarcir la forma configurada (entropía negativa) pocas veces son efectivas. En otros términos, se identifica en el territorio y la ciudad un escenario de desorden, con frecuentes pugnas entre fuerzas denominadas entrópicas y negentrópicas (González, 2017).

Asumimos que la forma de la ciudad no es constante, no lo ha sido nunca, sino que mucho depende del ejercicio de vivir la ciudad. Aunque aquí 
se identifican las facilidades de acceso a un conjunto diverso de productos y servicios que conlleva el que las personas experimentemos los beneficios de la vida urbana. No obstante, en este espacio también se experimentan diversos inconvenientes, relacionados, algunos de ellos, con la práctica de la movilidad. Sobre todo, porque asignamos tiempos importantes para los desplazamientos intraurbanos cotidianos. Asimismo, quienes vivimos en ciudad, experimentamos también el costo de la vida urbana (González, 2017).

En este marco, nuestro trabajo se supedita a los estudios sobre la movilidad y los sistemas de transporte, y pretende la implementación de los preceptos de la teoría de los sistemas en los procesos de planificación del espacio urbano y periurbano, respectivamente. La preocupación estriba hacia la gestación y desarrollo de hibridaciones morfológicas no consideradas inicialmente en la práctica de la movilidad urbana no sistémica. Sobre todo porque actualmente el consumo desmedido del espacio físico y de los medios para moverse en ese espacio cuestionan la sostenibilidad de las ciudades en el futuro inmediato. El objetivo central revisa la inequidad en el acceso a las infraestructuras para la movilidad urbana que existe en la periurbanización sureste del municipio de Tonalá, Jalisco, dentro del Área Metropolitana de Guadalajara, en México. Esto en función de observar inicialmente las condiciones físicas de las infraestructuras, y suponer que la entidad reguladora de la movilidad motorizada e individual genera entropía en la ciudad-sistema, y sus alternativas se ven rebasadas en el corto tiempo, pues no atienden el problema en su conjunto, sino que se limitan a solucionar puntualidades en el espacio físico. Estas intervenciones podríamos llamarlas como alternativas-parche efectuadas generalmente a posteriori. Si bien pretenden subsanar las omisiones en cuanto a la dotación, tanto de infraestructura vial como de servicios básicos, se convierten en una serie de remiendos o adecuaciones compensatorias en materia de satisfacción de las necesidades sociales (González, 2017).

Metodológicamente, hemos realizado un ejercicio cuasi-etnográfico a través de diálogos abiertos, en virtud de que no siempre es posible considerar tiempos prolongados para el proceso de indagación (Jeffrey \& Troman, 2004). De ahí la complejidad que nos presentan las investigaciones etnográficas tradicionales, identificadas por tiempos prolongados y bajo el argumento de que mayores tiempos destinados al trabajo de campo implican, necesariamente, mayores y mejores comprensiones del fenómeno estudiado y, consecuentemente, de sus resultados. Sin embargo, en los ejercicios cuasi-etnográficos, el tiempo invertido en el trabajo de campo suele ser menor (Silva \& Burgos, 2011). De igual forma, nos hemos auxiliado del análisis de contenidos temáticos, mediante la revisión de registros documentales, fotográficos e imágenes satelitales, los cuales nos han permitido, mediante el análisis y discusión correspondiente, el vertido de inferencias.

\section{Infraestructuras de transporte y el ejercicio de la movilidad urbana motorizada}

En la actualidad, las infraestructuras de transporte son construidas y reconstruidas en función del paradigma en términos kuhnianos de la movilidad motorizada. En efecto, se han diversificado algunas consideraciones con estímulos para el uso del autotransporte colectivo, y con restricciones y cargos por el uso del vehículo particular. Sobre todo porque las políticas energéticas en materia de combustibles en muchos países han configurado escenarios complicados para el usuario del vehículo privado, toda vez que estos operan con cierta volatilidad y acordes con los vaivenes tarifarios internacionales. No obstante, se sigue incentivando la industria automotriz y se promociona consistentemente el imaginario social sobre la posesión vehicular como símbolo de independencia y libertad, poder adquisitivo y status social, comodidad y confort. Este paradigma ya ha sido estudiado y analizado bajo la teoría de las representaciones sociales que guían la acción del individuo, que Maxwell (2001) denominó repertorios discursivos. Estos han permitido conocer las opiniones y justificaciones de la gente, cuando recurren a emplear discursos a favor de la utilización del coche, para sostener amplios ideales sociales a través de estrategias que implican delegar en otros la responsabilidad de las externalidades que genera este modo de transporte. Efectivamente, para este autor, los significados positivos de la utilización del coche asociado con el ahorro, ir de compras, trabajo, relaciones sociales, familiares, amigos y vecinos, conviven con los aspectos más problemáticos que resultan de las consecuencias sociales y ambientales de los crecientes niveles de propiedad y uso masivo de modos de transporte individual motorizado, los cuales 
van en contravía de una movilidad urbana sostenible. Esta dependencia del uso intensivo de modos de transporte motorizado individual (carros y motos), junto con las externalidades negativas, tales como contaminación atmosférica, congestión y accidentes de tránsito, dejan altos costos socio-económicos y de tiempo que al final deben ser asumidos por la sociedad en su conjunto (Miralles \& Cebollada, 2003).

Si partimos del supuesto en torno a que las infraestructuras para la movilidad urbana son ejes fundamentales que coadyuvan con el bienestar colectivo de las personas que habitan el sistema urbano y sus franjas periurbanas. Es decir, entre el entorno y el sistema, donde se perciben con mayor claridad las formas físicas de uno y otro, debido a los intercambios termodinámicos. Entonces, desde ese supuesto, resulta plausible tener infraestructuras adecuadas que permitan una movilidad ambientalmente sostenible, económicamente eficiente y socialmente incluyente en la urbanización y más allá. Sin embargo, los estudios sostienen que en este más allá (periferia) se identifican sobredosis de inequidad en la accesibilidad a los bienes y servicios del Estado, y a las oportunidades que brindan las ciudades para quienes habitan en ellas (Lupano \& Sánchez, 2009). Esta distribución inequitativa en el acceso a las infraestructuras para la movilidad y medios de transporte en el espacio intraurbano y periurbano, se transforma en desigualdades en el acceso a los servicios de la ciudad, así como en los costos y beneficios de todos los actores de la movilidad en su conjunto (Alcántara, 2010).

Así pues, las infraestructuras para la movilidad son un elemento determinante que influye de alguna manera en la definición del modelo de ciudad que se quiera tener. En las últimas décadas, varias ciudades latinoamericanas optaron por el abandono de sus centros y apostaron por la conurbación de la periferia, lo que ha incentivado el modo de transporte privado como principal alternativa de movilidad para realizar los desplazamientos con todas las externalidades negativas que este modelo de ciudad expandida ha generado (Miralles, 2013). En este tópico, para que la ciudad cumpla su función esencial, se requiere garantizar condiciones básicas de habitabilidad, accesibilidad y movilidad en sus diferentes actividades cotidianas. Esto porque, al no proveerlos o proveerlos deficientemente, se están generando escenarios entrópicos, o bien, se está trabajando para situaciones caóticas. Tan solo en el caso de las infraestructuras para la movilidad, las desventajas y los costos en términos económicos y sociales han sido enormes, impactando de manera negativa el bienestar colectivo y debilitándose la transformación del hábitat en los espacios periurbanos (Mignot et al., 2010).

En este contexto, la red de transporte es considerada como una malla de canales integrados por donde se transportan los flujos de usuarios desde sus orígenes y sus destinos, buscando que este ejercicio ocurra de una manera eficiente y conveniente, no solo para ellos sino para la ciudad. Sin embargo, esto solo podrá conformarse si la red es concebida bajo una visón sistémica (Mundó, 2002, p. 291).

[...] la planeación debe ser una actividad continua, que acompañe la evolución del sistema estudiado, así como la naturaleza de sus problemas y la eficacia de las soluciones adoptadas (Sedesol, 2001, p. 3).

Entropía en la periurbanización de la ciudadsistema: la triada velocidad, distancia y tiempo

El concepto entropía ha sido abordado desde diversas aristas de las ciencias físicas y sociales. Se le relaciona muchas veces con la energía; como una forma de medición de esta, particularmente la no utilizada por el sistema para la generación de trabajo. Aunque el concepto también puede ser entendido como un estado de desorden que experimentan los sistemas. Si combinamos este concepto con el término de fuerza, tenemos entonces sistemas en los que pueden presentarse conjuntos de fuerzas de carácter entrópico que lo llevarían al caos. Estas fuerzas no necesariamente serían internas (esfuerzos), sino también externas (cargas) que vendrían del entorno. Entre ellas ocurre, como se ha dicho en otras ocasiones, un intercambio de flujos de materia y energía aún no delimitados temporalmente entre ambas categorías, y la entropía no estaría relacionada solamente con los esfuerzos o con las cargas. En este sentido, al menos en los sistemas sociales, se identifican pugnas entre fuerzas entrópicas y neguentrópicas externas-internas, internas-internas y externas-externas. En suma, la situación que experimentan las ciudades respecto a sus formas puede ser estudiada por sus niveles de entropía. Aquí, la neguentropía o entropía negativa sería la encargada de subsanar ese desorden termodinámico y de mantener, de alguna forma, la homeostasis 
del sistema o, en otra conceptualización, un estado uniforme. En el estado uniforme, los sistemas logran evitar el incremento de entropía y se hace posible la generación de ciertos estados de orden y organización crecientes (Bertalanffy, 1976). En este sentido, la planificación urbana y territorial desde el enfoque de los sistemas pudiera representar esa entropía negativa necesaria en la ciudad sistema.

En materia de transporte, desde hace bastante tiempo, los términos velocidad, distancia y tiempo han generado controversia teórica en su práctica espacio-geográfica. Sobre todo porque para el incremento o decremento de alguno, necesariamente se tiene que modificar la magnitud del otro. En esta ecuación, tal parece que la variable tiempo cobra una relevancia particular; y es que, desde hace miles de años, el hombre ha buscado incrementar la velocidad en el desplazamiento a través de mejoras en los medios de transporte o en la infraestructura para la movilidad. Primeramente, el invento de la rueda marcó un parteaguas en la antigüedad, el desplazamiento mediante la fuerza motriz de los équidos (burros, mulas, caballos) posteriormente el agua, los minerales, hasta llegar a los hidrocarburos. Asimismo, la infraestructura también ha experimentado cambios significativos; los caminos de brechas y veredas pasaron a los de terracería, adoquinado, empedrado, concreto hidráulico y asfáltico, respectivamente. A la utilización de estos materiales se le ha sumado el diseño y el mejoramiento geométrico del trazo vial; la experiencia ha permitido reducir los tramos con curvaturas complejas, se ha introducido señalética e instrumentos formales de regulación del tránsito, los cuales paulatinamente se han ido ajustando acordes con las exigencias y marcos regulatorios locales e internacionales.

En el ámbito intraurbano, la velocidad está sujeta a diversos factores tales como: a) el medio de transporte utilizado para el desplazamiento (a pie, bicicleta, motocicleta, autotransporte privado, autotransporte público, otros), b) la hora en la que se ejecuta el desplazamiento (horas pico, horas valle) y el día (entre semana, fin de semana), c) la vía utilizada para el desplazamiento (vía rápida, vía principal, vía secundaría, etc.), y d) el tipo de espacio transitado (zona escolar, zona comercial, zona residencial, etc.). Efectivamente, la distancia euclidea no cambia de posición. Sin embargo, sí cambia el tiempo, el cual varía en función de la velocidad y la posición respectivamente (González, 2017). Asimismo, desde los albores de la historia, el tiempo haya sido una variable por minimizar en los trayectos cotidianos. Pues se busca aprovecharlo al máximo, o gastarlo lo menos posible en la movilidad cotidiana; so pena que un mayor tiempo en el desplazamiento implica necesariamente mayores costos económicos, sociales y ambientales. Sí, efectivamente, en las ciudades entrópicas el ejercicio de la movilidad motorizada demanda cuantiosas inversiones de materia, energía y tiempo, e implica importantes costos para la construcción de grandes infraestructuras viarias como puentes, carreteras, viaductos, deprimidos, autopistas de alta velocidad y otras infraestructuras que se construyen para maravillar o colonizar (Larkin, 2008). Por su parte, las inversiones para los modos de transporte sostenible, tales como caminar, desplazarse en bicicleta o en transporte público sustentable, distan enormemente de la asignación de recursos en los presupuestos municipales y en otros niveles presupuestarios de gobierno; a tal grado que un $80 \%$ se destina para obras viales que privilegian al carro particular (CEJ, 2013).

\section{El acceso a las infraestructuras para la movilidad en Tonalá, México}

La población del Área Metropolitana de Guadalajara (AMG) cuenta con 4.865.122 habitantes y representa el $62,02 \%$ del total del estado de Jalisco, siendo la segunda más grande de México (INEGI, 2015). El municipio de Guadalajara es el de mayor población $(30,01 \%)$, pero el de menor superficie, con $151,4 \mathrm{~km}^{2}$; por tanto, es el más denso, con cerca de 9.644,31 hab $/ \mathrm{km}^{2}$. Tonalá, ubicado en la parte oriental del AMG, con una población de 478.689 habitantes $(10,64 \%)$ y densidad de $2.881,9 \mathrm{hab} / \mathrm{km}^{2}$, es la tercera municipalidad con la densidad más alta después de San Pedro Tlaquepaque. De aquí, doce colonias circunscritas en el área de estudio: Santa Paula, Santa Isabel I y II, Santa Rosa, Hacienda Real, Arroyo de en Medio, Las Puertas, La Ladrillera, Jauja, La Rusia, Alfarera, Guadalupana y La Punta tienen una población de 46.357 habitantes. Es decir, representan el 8,64\% del total de la población del municipio (IIEG, 2010).

Las personas que residen en estas colonias habitan viviendas constituidas en su mayoría mediante el proceso de autoconstrucción, carente de 
infraestructura pública básica, principalmente de tipo hidrosanitaria y de transporte. Este tipo de vivienda se engloba en la categoría denominada por Giglia (2012) como habitar-progresivo y, normalmente, ha ido acompañándose (sin mezclarse al menos de inicio) con la vivienda en serie; el best-seller de la industria inmobiliaria, la cual ha ofertado vivienda encofrada en un medio físico delimitado perimetralmente y dando pauta a formas complejas de configuraciones espaciales posteriores; no en términos deterministas, sino más bien en términos probabilísticos. Esta vivienda en serie (de acuerdo con la misma autora), cumple con la categoría del habitar-racional. Sin embargo, existe una tercera forma de habitar el espacio que resulta de una hibridación entre la segunda y la primera. Es decir, vivienda racional que va modificando su fisonomía en el tiempo, a través de mejoras manifiestas en ampliaciones y remodelaciones; una especie de combinado denominado por Barranco \& González (2016) como habitar racional-progresivo.

La llegada del habitar racional (inputs), en conjunción con el existente (habitar progresivo), fue configurando formas diferentes (outputs) de habitar el espacio periurbano y urbano, respectivamente. Estas formas no necesariamente han sido hostiles respecto a las previamente existentes, debido a que ya existía entropía manifiesta en la generalidad de la carencia (uniformidad matizada) de los servicios básicos. No obstante, una vez intervenido el espacio físico, se presentan diferencias infraestructurales con mejoras de accesibilidad-laterales para el habitante inicial. En estas mejoras laterales, el beneficio incipiente, insuficiente o deficiente ha ocurrido indirectamente, y el escenario para el acceso a la infraestructura del habitar progresivo dista significativamente respecto a los del habitar racional. En resumen, se perciben escenarios inequitativos en el proceso de la movilidad urbana y periurbana. Esta inequidad es identificada en las infraestructuras de transporte destinadas, tal parece, para incentivar el uso del coche particular y, en consecuencia, para habitantes con ingresos que les permitan sostener este medio de transporte.

El escenario de estas clases sociales, denominadas por Bauman (2010) como clases turistas, tiene unos altos patrones de movilidad a nivel local y global. De igual forma, justifican sus patrones de alta movilidad dependientes del coche, y creando historias estructurales, para conocer hábitos en el transporte y actitudes por medio del relato o narración de los individuos en la elección de su modo de transporte público o privado (Freudendal, 2009). Estas historias permiten que los individuos justifiquen el uso del automóvil, a pesar de ser conscientes de las externalidades y consecuencias negativas que este genera al medio ambiente natural y a la población en su conjunto, y sostienen el argumento que "[...] el coche es como el pegamento de la vida cotidiana y sin el coche la vida cotidiana no puede ser coherente", o bien, "[...] cuando uno tiene hijos uno necesita un automóvil, el tren siempre está retrasado y el auto como posibilidad de creación". Estas exteriorizaciones ven en el carro la única opción que le garantiza diferentes alternativas de movilidad que ningún otro modo de transporte ofrece, especialmente la sensación de libertad de moverse para diferentes destinos o de cambiar de ruta cuantas veces se quiera (Freudendal, 2009).

\section{Metodología}

Metodológicamente se realizó un ejercicio cuasi-etnográfico como forma básica para entender, describir y comprender el tema. Esto se hizo mediante diálogos abiertos auxiliados por la observación participante (Ameigeira, 2007). Asimismo, auxiliados con los registros fotográficos e imágenes de Google Earth, fue posible determinar, por ejemplo, que en la colonia Hacienda Real conformada por dos etapas (3070 unidades habitacionales para la primera y 1500 unidades para la segunda), el porcentaje de habitabilidad promedio (casas ocupadas) es del 70\%, lo que significa que alrededor de 3199 viviendas se encuentran habitadas y de ellas el 95\% tiene en promedio 2 vehículos, según datos suministrado por la Administración de los Fraccionamientos Cerrados.

En función de lo anterior, hay más vehículos que viviendas ocupadas; situación que refleja la fuerte tendencia del uso del carro particular de las personas que habitan los fraccionamientos cerrados de esta parte del municipio. Este paradigma de movilidad dependiente del carro particular presente en la colonia Hacienda Real, contrasta con los modos de transporte utilizados por la mayoría de los habitantes que habitan las otras colonias elegidas selectivamente, las cuales utilizan con mayor frecuencia el autotransporte público. Sin embargo, las condiciones físicas de la infraestructura para la accesibilidad aumentan indudablemente los tiempos del desplazamiento (Figura 1 y Figura 2). 
En la actualidad existen nueve rutas de transporte público que ofrecen servicio en la porción estudiada: R-615, R-615A, R-614, R-644 B (Carril y Jauja), R-03, R-59B y R-178 vía 1 y vía 2. Estas conectan la periurbanización con el centro metropolitano y han tenido que interactuar con otro modo de transporte motorizado denominado "moto taxi"; un sistema irregular, de acuerdo con la autoridad, que, sin embargo, ha permitido cubrir las distancias que el autotransporte público se ve impedido, toda vez que existen restricciones para el acceso al interior de los fraccionamientos. Asimismo, el auge de este medio de transporte se debe también a la demora, baja frecuencia y desorganización del subsistema de

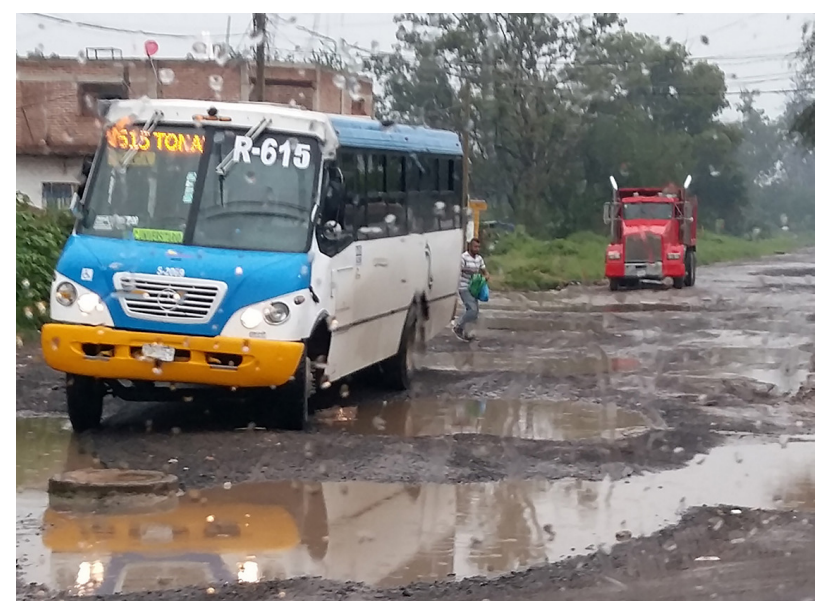

Figura 1 - Infraestructura de transporte en la colonia Jauja, Tonalá-Jalisco Fuente: Elaboración propia.

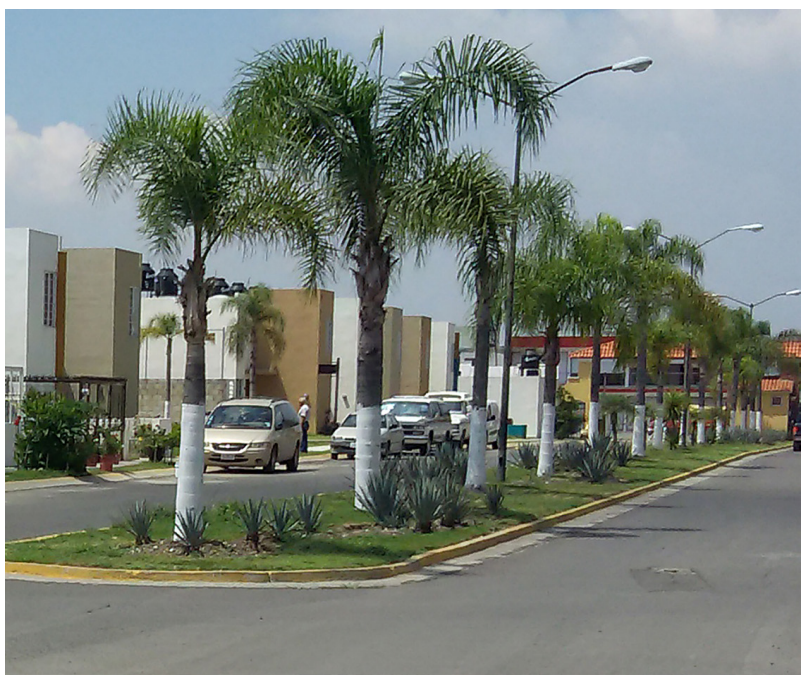

Figura 2 - Infraestructura de transporte en la colonia Hacienda Real, Tonalá-Jalisco Fuente: Elaboración propia. transporte. Sobre todo porque, para el Observatorio de Movilidad, el transporte público del AMG carece de una infraestructura adecuada y una red lo suficientemente amplia, extendida, eficiente y de calidad (Sánchez, 2015).

Las deficiencias físicas de las infraestructuras para la movilidad son evidentes en la mayoría de las colonias del sureste del municipio, lo que ha incentivado la utilización de la bicicleta y la motocicleta como medidas que les permiten solventar el desplazamiento, con todo y a pesar de los riesgos de accidentalidad que este modo de transporte implica. Toda vez que no existen las adecuaciones viarias pertinentes para el tránsito de medios de transporte no motorizados (Figura 3).

Las infraestructuras que se construyen no son extensivas y no se visualizan en algunas de las colonias de la periurbanización, donde se encuentra asentadas buena parte de las clases trabajadoras denominadas por Bauman (2010) vagabundos, debido a que viven en un espacio pesado, en condiciones socioeconómicas complicadas, donde "[...] solo se ve pasar el tiempo y nunca pasa nada". En este sentido, aleatoriamente fueron elegidos habitantes para entablar diálogos abiertos sobre la experiencia vivida en el espacio habitado. En el diálogo abierto con María; una trabajadora independiente y habitante de la colonia La Ladrillera desde hace más de 20 años, comenta que

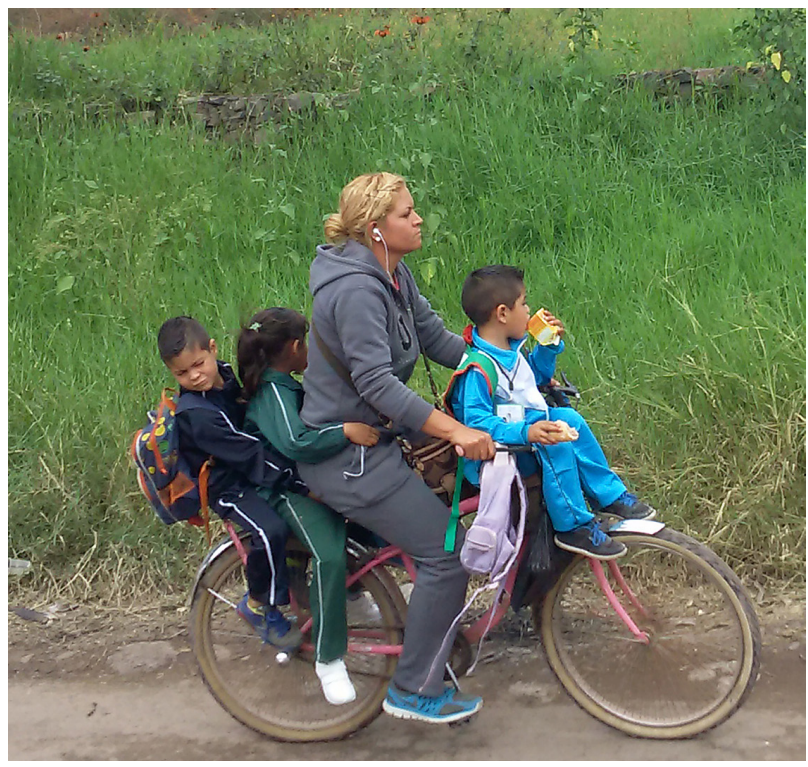

Figura 3 - Infraestructura existente para la movilidad sostenible, Colonia Javia y Hacienda real, agosto 2016

Fuente: Elaboración propia. 
[...] para la construcción de los fraccionamientoso coto cerrados muchas comunidades de las colonias como La Ladrillera, Las Puertas y Arroyo de en Medio, fueron desplazadas y marginadas, por los constructores y urbanizadores inmobiliarios comprándoles sus prediosy terrenos a bajos costos, con la promesa de mejorarles y proveerles redes de abastecimiento de agua y saneamiento, vías pavimentadas pero esas obras solo las construyeron para los que habitan en los fraccionamientos o cotos cerrados (Diálogo con María Jiménez, agosto 2016).

Efectivamente, las personas de bajos ingresos que viven en la periurbanización mantienen aspiraciones territorializadas de lograr algún día el sueño de “[...] tener casa propia y así compensar lo perdido en otros ámbitos de la vida y resarcir anteriores carencias de lo que nunca se tuvo" (Hiernaux \& Lindon, 2004). De lograrse el sueño de obtener una casa propia o el predio correspondiente en la periurbanización, estos no cuentan en la mayoría de los casos con la infraestructura y los equipamientos básicos como agua potable, saneamiento y rutas de transporte, por tratarse de asentamientos fuera de los límites considerados por los deficientes planes y programas de planificación urbana, o porque contienen irregularidades en la adquisición de la propiedad de la tierra (Romero, 2014). En este sentido, en diálogo abierto realizado con José; una persona soltera, activista ambiental y estudiante universitario, quien reside en la colonia de Santa Isabel, comparte que

[...] las empresas de servicios públicos especialmente de agua y gas natural, en muchos casos les niegan a los moradores la conexión e instalación de dichos servicios, con el argumento de que estas familias no tienen capacidad económica para pagar la factura (Diálogo con José Iglesias, septiembre 2016).

En resumen, las personas que tienen mayores ingresos son los que gozan del privilegio de contar y disfrutar de estos servicios (Lorrain, 2005; Anand, 2011; Kooy \& Bakker, 2008).

Estas realidades evidencian en una misma porción geográfica, diferencias en la sociedad del riesgo móvil, que vivimos en esta época de postmodernidad, también llamada sociedad tecnológica, donde las estructuras sociales se vuelven inestables y permeables (Kesselring, 2008). Por ejemplo Icel; una mujer que trabaja en un local de venta de celulares, soltera, perteneciente al grupo de personas con restricciones en su movilidad y que vive con sus padres en la colonia Las Puertas (una colonia contigua a los fraccionamientos cerrados de la colonia Hacienda real), expresa que

[...] en la colonia donde vivo cuando llueve la calle se vuelve un lodazal (suelo fangoso), cuando es verano es un polvero (exceso de polvo) y a la mayoría de las personas nos toca caminar varias cuadras en medio del lodo o el polvo, para llegar a la vía por donde pasan los camiones (autobuses) (Diálogo con Icel Rocha, agosto 2016).

Estas razones de inestabilidad y permeabilidad conducen al fenómeno de la exclusión social, el cual margina y limita el goce de garantías a sectores de la población.

De acuerdo con la SEU (2003), las personas que experimentan exclusión social en zonas periurbanas tienen cinco tipos de barreras para llegar a los principales servicios de disponibilidad y accesibilidad física de transporte: la disponibilidad de transporte y su accesibilidad física, la seguridad de los transportes y el costo, los horizontes de viaje limitados, la ubicación de los servicios y frecuencia del transporte público, la fiabilidad y la cobertura de la red. Estas barreras se han observado en las colonias que comprenden la porción espacial del estudio, con excepción de los que viven en los fraccionamientos cerrados. Aquí, como lo comenta Judith González; una residente casada, madre de cuatro hijos, trabajadora independiente que lleva viviendo varios años en esta colonia, comparte:

[...] mi único modo de transporte es el carro, porque me muevo y voy para donde quiero con rapidez, tengo mayor comodidad y seguridad, me gusta conducir, sentir la velocidad, la libertad, además con hijos es muy complicado andar en camión (autobús). El transporte público es muy demorado, no es seguro, es incómodo y uno va como sardina (Dialogo con Judith González, noviembre 8 de 2016).

Para evidenciar la inequidad de la infraestructura de la movilidad urbana que existe en la porción bajo estudio, se parte del hecho de que técnicamente el carácter de una red viaria en la metrópoli significa que la distancia y el acceso son a menudo definidas en gran parte por la longitud y la topología de puntos de conexión de dicha redes (Graham, 2000). En este sentido, en diálogos con Esmeralda; una profesional en administración, casada, madre de dos hijos mayores 
de edad, residente de la colonia y administradora de la segunda etapa, expresa:

[...] uso el transporte público de manera esporádica, las rutas pasan enfrente del fraccionamiento pero los camiones andan saturados, se demoran mucho en pasar entre 30 a 40 minutos y no hay respeto por parte de los conductores, ellos andan a altas velocidades, por ello prefiero usar casi siempre mi carro (Dialogo con Esmeralda Aldana, noviembre 20 de 2016).

Así pues, el acceso a las rutas de transporte público determinado en función de las distancias promedio de caminata y estado físico de la vía, desde un punto equidistante de cada colonia respecto a los principales ejes viales como Arroyo de en Medio, Carretera libre de Zapotlanejo, Vía del Carril, José Álvarez-Lázaro Cárdenas y Las Trojes (ejes principales por donde circulan las rutas de transporte público y demás modos de transporte), condujo mediante la inspección visual a la determinación de diferentes escalas de calificación que, de acuerdo con el IRAP (2015), las infraestructuras viales tienen diferentes atributos, en los cuales el estado físico del pavimento se puede evaluar en tres categorías: bueno, regular y malo.

De manera similar a la valoración anterior, el IDU (2013) de la ciudad de Bogotá utiliza, para evaluar el estado de conservación de su infraestructura vial urbana, tres categorías: un estado de la infraestructura viaria considerado como malo, el cual engloba las infraestructuras que se encuentran sin pavimentar, normalmente con baches, huecos y, en la mayoría de casos, sin redes de drenajes para la evacuación de las aguas superficiales; lo que dificulta la movilidad para todos sus usuarios. En tanto que se consideran infraestructuras en estado regular aquellas que están empedradas o pavimentadas, pero que con el tiempo y falta de mantenimiento se han deteriorado y requieren de una reconstrucción total. Finalmente, las infraestructuras consideradas como buenas son las compuestas por vías pavimentadas bajo especificaciones técnicas constructivas de buena calidad; estas cuentan con obras de drenajes, señalización, andadores, mantenimiento permanente y elementos que permiten la movilidad a los diferentes usuarios, de manera segura e incluyente.

En el caso de México, se tienen infraestructuras clasificadas conforme a su carpeta de rodamiento en tres grupos principalmente. Las infraestructuras pavimentadas circunscriben a los caminos hechos sobre la base de un revestimiento, con materiales resistentes para el tránsito de vehículos pesados y con una superficie tersa de rodamiento de asfalto. En tanto que las infraestructuras revestidas conjuntan caminos de una capa de materiales seleccionados, extendidos y compactados sobre las terracerías, para recibir las cargas que produce el tránsito. Y finalmente, las infraestructuras viales de terracería que agrupan los caminos acondicionados con materiales naturales como piedra bola, tezontle y otros, para el tránsito de vehículos y/o personas (SCT, 2010). En este sentido, es conocido que mediante la carpeta de rodamiento es posible evaluar el grado de accesibilidad, sobre todo, porque esta puede ser entendida como la facilidad para viajar a cada unidad espacial dentro del sistema territorial. Es decir, la capacidad para poder llegar a ciertos lugares en un tiempo y con un esfuerzo razonable (Santos \& De las Rivas, 2008).

\section{Resultados}

Apoyados en la observación participante y la tecnología de Google Earth se determinaron las distancias geométricas promedio de caminata, para acceder a las rutas de autobuses en cada una de las doce colonias estudiadas (Tabla 1 ).

Como se puede observar, en la mayoría de las colonias los habitantes deben caminar una distancia euclidea promedio mayor de $500 \mathrm{~m}$. Es decir, el límite superior de la distancia máxima sugerida para acceder a una ruta de transporte público según estándares internacionales (ITDP, 2014). En este contexto, se evidencia que las colonias que tienen mejores infraestructuras (físicamente) son aquellas en las que se realizan menos distancias geométricas de caminata para llegar a una ruta de transporte y por tanto, con menor tiempo del recorrido, mientras que las que tienen un mal estado físico de la infraestructura viaria, se ven obligados a caminar mayores distancias geométricas y consecuentemente, con mayor tiempo en sus traslados, como es el caso de las colonias La Rusia, Santa Rosa, Las Puertas y La ladrillera. Así mismo, el estudio muestra que 7 de las 12 colonias estudiadas tienen distancias euclidianas de caminatas superiores a los máximos establecidos a nivel internacional, lo que se traduce, como lo hemos comentado, en un mayor tiempo para el desplazamiento entre la 
Tabla 1 - Distancias promedios de caminata a una ruta de transporte y estado de la infraestructura vial

\begin{tabular}{cccc}
\hline$N^{\circ}$ & Nombre Colonia & $\begin{array}{c}\text { Distancia de } \\
\text { caminata promedio } \\
\text { al Transporte } \\
\text { Público (metros) }\end{array}$ & $\begin{array}{c}\text { Estado de la } \\
\text { Infraestructura } \\
\text { vial para la } \\
\text { movilidad urbana }\end{array}$ \\
\hline 1 & Santa Paula & 450 & Regular \\
2 & Santa Isabel I y II & 545 & Mala \\
3 & Santa Rosa & 832 & Mala \\
4 & Hacienda Real & 100 & Buena \\
5 & Arroyo de En medio & 562 & Regular \\
6 & Las Puertas & 755 & Mala \\
7 & La Ladrillera & 628 & Mala \\
8 & Jauja & 496 & Regular \\
9 & La Rusia & 1059 & Mala \\
10 & Alfarera & 185 & Mala \\
11 & Guadalupana & 590 & Regular \\
12 & La Punta & 120 & Buena \\
\hline
\end{tabular}

Fuente: Elaboración propia, con base en la observación participante y Google Earth (2016).

vivienda y la parada del autobús. A este tiempo, hay que adherirle el tiempo de la espera de la unidad del transporte (frecuencia de paso) y el tiempo del trayecto (Figura 4). En suma, esta entropía complica el ejercicio de la movilidad cotidiana.

En función de las pruebas encontradas, tanto en la fisonomía de las infraestructuras como en la organización de los subsistemas de movilidad motorizada, resulta plausible identificar diferencias significativas en la práctica de movilidad urbana. Sobre todo porque las colonias que cuentan con mejores condiciones físicas en sus infraestructuras viarias experimentan menor entropía en el acceso a la red vial, independientemente del medio de transporte utilizado. Es decir, aunque hay diferencias notorias en tiempo, existen similitud de condiciones para la accesibilidad; por ejemplo, solo en la colonia Haciendan Real, 77,7\% de los diferentes servicios de transporte público que se ofertan pasan a una distancia promedio de 100 metros de las viviendas. Sin embargo, cuando el escenario anterior se caracteriza por mayores distancias para acceder a las rutas de transporte aunadas a una o dos rutas (aproximadamente el 22\% para este ejemplo), ocurren escenarios entrópicos.

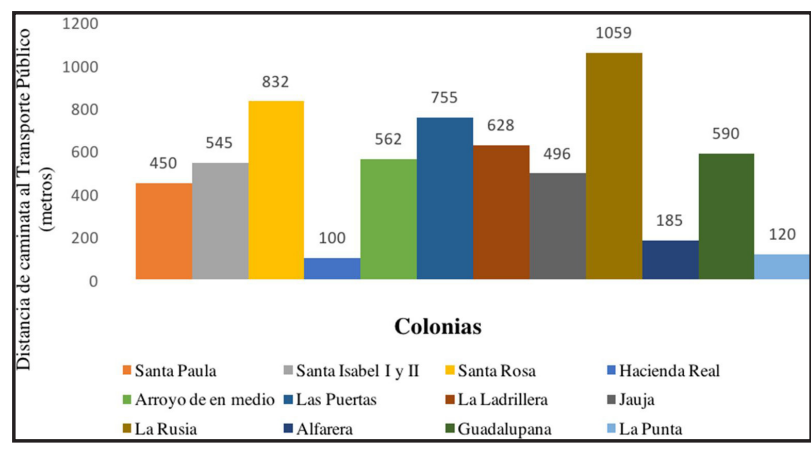

Figura 4 - Distancia promedio de caminata hasta una ruta de transporte público en cada colonia Fuente: Elaboración propia.

\section{Conclusiones}

Para Beard et al. (2016), las ciudades que vienen experimentando crecimientos acelerados de urbanización en sus periferias deben encontrar alternativas de mejorar e incrementar su infraestructura de servicios públicos (acueductos, alcantarillado, movilidad, otras), y convertirse en ciudades con equidad y calidad de vida. Este cambio de paradigma permitirá volver a las ciudades del siglo XIX, llamadas por Lorrain (2005) las polis o ciudad de peatones, las cuales proporcionan mayores proximidades, ahorros de tiempos de viaje, accesibilidad y mezcla de usos del suelo (Miralles \& Marquet, 2014). También es posible garantizar una mejor conectividad entre los bienes y servicios de la ciudad, menor dependencia de modos motorizados, disminución de la contaminación ambiental y, consecuentemente, mejores condiciones de salud pública.

La inequidad en las infraestructuras de transporte para la movilidad urbana es un fenómeno presente y complejo con mayor evidencia en la periurbanización metropolitana; aunque no es exclusiva de esta. De ahí que la prospectiva de garantizar buenas infraestructuras para la movilidad sea vista como el principal desafío que le depara a las ciudades. En este sentido, la nueva agenda urbana Habitat III, aprobada recientemente en Quito, Ecuador en octubre de 2016, exhorta a los gobiernos a nivel global a construir entornos urbanos incluyentes, sostenibles, compactos, seguros y resilientes a las amenazas por riesgos naturales (ONU, 2016).

Teniendo en cuenta que en las colonias estudiadas existen altas movilidades en los diferentes modos de transporte, es posible tener una mejor calidad de vida, siempre y cuando se mejoren sus infraestructuras de 
transporte. A pesar de que existe una gran cantidad de personas que se movilizan en modos no motorizados, la infraestructura existente no les garantiza moverse de manera segura y confortable. En ese sentido, se hace pertinente implementar en estas colonias el concepto de calles completas, que muchas ciudades del mundo han empezado a apropiárselo, tanto para la adecuación de sus infraestructuras urbanas como para la construcción de las nuevas, donde se le da prioridad a modos de transporte no motorizados, sin ser excluyente con los otros modos motorizados individuales y colectivos (Smart Growth America, 2015).

En la periurbanización del sureste del municipio de Tonalá, existen infraestructuras consideradas de primera, con las mejores condiciones constructivas y de mantenimiento; pero también existen infraestructuras de tercera, con todas las precariedades y deficiencias físicas. Las primeras cuentan con altos estándares de servicios infraestructurales de transporte y las restantes se ven sometidas a condiciones infraestructurales de mal estado y sin la posibilidad de que el usuario que se encuentra fuera de los intervalos recomendados de caminata pueda reducir sus distancias geométricas y consecuentemente, sus tiempos para acceder a los servicios de transporte. En síntesis, el escenario que experimenta el ejercicio de la movilidad y sus principales actores se torna entrópico, con diferentes desordenes en la forma física, que configuran nuevas formas aún más complejas, donde los flujos de materia y energía están generando no solo caos vial, sino también tiempos muertos y efectos socioeconómicos y ambientales importantes. La concepción del sistema-ciudad y el subsistema transporte, tal parece que son concebidos como de tipo cerrado por la planificación. Sin embargo, la evidencia refuerza la tesis acerca de que la ciudad y sus componentes son sistemas abiertos y en este sentido, el proceso de planificación urbano-territorial puede representar la fuerza neguentrópica con potencial regulador del conflicto termodinámico originado por las actuaciones en el espacio físico relacionadas con la práctica de vivir la ciudad.

\section{Referencias}

Alcántara, E. V. (2010). Análisis de la movilidad urbana: espacio, medio ambiente y equidad. Colombia: CAF. Recuperado el 03 febrero de 2017, de https://www.caf. com/media/3155/An\%C3\%A1lisis_movilidad_urbana.pdf
Ameigeira, A. R. (2007). El abordaje etnográfico en la investigación social. Vasilachis de Gialdino, I. (coord). Estrategias de investigación cualitativa (pp. 107-151). Barcelona: Gedisa.

Anand, N. (2011). Pressure: the politechnics of water supply in Mumbay. Cultural Anthropology, 26(4), 542-564. http://dx.doi.org/10.1111/j.1548-1360.2011.01111.x. PMid:22171410.

Barranco, M. C., \& González, M. G. (2016). El transporte intracondominal en la movilidad cotidiana de la periurbanización: el enlace comunitario del Área Metropolitana de Guadalajara. Revista Transporte y Territorio, 14, 167-188. Recuperado el 12 de septiembre de 2016, de http://revistascientificas. filo.uba .ar/ index.php/rtt/article/view/2434/2092

Bauman, Z. (2010). La globalización: consecuencias humanas. México: Fondo de Cultura Económica.

Beard, V.A., Mahendra, A. \& Westphal M.I. (2016). Towards a more equal city: framing the challenges and opportunities. Washington: World Resources Institute. Recuperado el 4 de noviembre de 2016, de http://www.wri.org/sites/ default/files/Towards_a_More_Equal_City_Framing_the_ Challenges_and_Opportunities_Exec_Summary.pdf

Colectivo Ecologista de Jalisco A. C. - CEJ. (2013). Costos económicos y ambientales de los autos en la área metropolitana de Guadalajara. México: LAC. Recuperado el 12 de noviembre de 2016, de http://ciudadpixel.mx/ documentos/Reporte\%20Autos\%20LAC\%20CEJ.pdf

Freudendal, M. P. (2009). Mobility in daily life: between freedom and unfreedom. USA: Farnham.

Giglia, A. (2012). El habitar y la cultura: perspectivas teóricas y de investigación. México: Anthropos.

González, M. (2017). Movilidad motorizada e infraestructuras de transporte en Culiacán: una situación entrópica. "Poder, Cultura y Desarrollo" (pp. 60-77). México: Universidad de Guanajuato.

Google Earth. (2016). Localización colonias sur-oriente Tonalá-Jalisco. Recuperado el 12 de diciembre de 2016, de https://earth.google.com/web/@20.58373198,103.24100503,1544.2151258a,3649.6679146d,3 $5 \mathrm{y},-0 \mathrm{~h}, 0 \mathrm{t}, 0 \mathrm{r}$

Graham, S. (2000). Cities and infrastructure networks. International Journal of Urban and Regional Research, 24(1), 114-119. Recuperado el 09 de mayo de 2016, de http:// onlinelibrary.wiley.com/doi/10.1111/1468-2427.00238/ 
epdf?r3_referer=wol\&tracking_action=preview_click\&show checkout=1\&purchase_referrer=www.google.com. mx\&purchase_site_license=LICENSE_DENIED

Hiernaux, D., \& Lindon, A. (2004). Desterritorialización y reterritorialización metropolitana: la Ciudad de México. Documents D'Anàlisi Geogràfica, 44, 71-88. Recuperado el 12 de junio de 2016, de http://ddd.uab.cat/pub/ dag/02121573n44/02121573n44p71.pdf

Institute for Transportation and Development Policy ITDP. (2014). TOP standard v 2.1 (Cap. 4., pp. 48-49). New York: EE.UU. Recuperado el 11 de mayo de 2016, de https://www.itdp.org/wp-content/uploads/2014/03/ DOT-Est\%C3\%A1ndar-2.1.pdf

Instituto de Desarrollo Urbano - IDU, Subdirección General de Desarrollo Urbano. (2013). Estado vial de la malla vial de Bogotá. Bogotá: IDU. Recuperado el 5 marzo de 2016, de https://www. idu.gov.co/documents/371801/425567/boletin_malla_vial_ bogota_24abr14.pdf/ef027911-20cd-468f-b02c-bf132af55991

Instituto de Información Estadística y Geográfica de Jalisco - IIEG. (2010). Información sociodemográfica por colonias. México: IIEG. Recuperado el 11 de mayo de 2016, de http://www.iieg.gob.mxgeneral.php?id=4\&idg=331

Instituto Nacional de Estadística, Geografía e Informática - INEGI. (2015). Pincipales resultados de la encuesta intercensal 2015 Jalisco. México: INEGI Recuperado el 11 de diciembre de 2016, de http://www3.inegi.org.mx/ sistemas/ biblioteca/ficha.aspx?upc $=702825079796$

International Road Assessment Programme - IRAP. (2015). Methodology fact sheet 3: road attributes. London: IRAP. Recuperado el 5 marzo de 2016, de https://www.irap. org/resources/\#mytabs|2

Jeffrey, B., \& Troman, G. (2004). Time for ethnography. British Educational Research Journal, 30(4), 535-548. http:// dx.doi.org/10.1080/0141192042000237220.

Kesselring, S. (2008). The mobile risk society. In W. Canzler, \& V. Kaufmann (Eds.), Tracing mobilities towards a cosmopolitan perspective (pp. 77-102). England: Aldershot.

Kooy, M., \& Bakker, K. (2008). Technologies of government: constituting subjectivities, spaces, and infrastructures in Colonial and Contemporary Jakarta. International Journal of Urban and Regional Research, 32(2), 375-391. Recuperado el 15 de diciembre de 2017, de https://www.researchgate. net/publication/4990998_Technologies_of_Government_ Constituting_Subjectivities_Spaces_and_Infrastructures_in Colonial_and_Contemporary_Jakarta
Larkin, B. (2008). Signal and noise: media, infrastructure and urban culture in Nigeria. London: Duke University Press. http://dx.doi.org/10.1215/9780822389316.

Lorrain, D. (2005). Gigacity: the rise or technological networks in daily life. In O. Coutard, R. Hanley \& R. Zimmerman (Eds.), Sustaining urban networks: the social diffusion of large technical systems. London: Routledge

Lupano, J. A., \& Sánchez, R. J. (2009). Políticas de movilidad urbana e infraestructura urbana de transporte. (Documento de proyecto Comisión Económica para América Latina y el Caribe - CEPAL). Santiago de Chile: Naciones Unidas. Recuperado el 17 de febrero de 2016, de http://repositorio. cepal.org/bitstream/handle/11362/3642/1/S2009021_es.pdf

Maxwell, S. (2001). Negotiations of car use in everyday life. In D. Miller (Eds.), Car cultures. Oxford: Berg.

Mignot, D., Aguiléra, A., Bloy, D., Cauble, D., \& Madre, J. L. (2010). Formas urbanas, movilidad y segregación. Revista Urban Public Economics Review, 12, 73-104. Recuperado el 14 de febrero de 2017, de http://www.redalyc.org/ articulo.oa?id=50414006003

Miralles, C. G. (2013). La movilidad, los transportes y el territorio. Un triángulo líquido y multiforme. In Anais del Seminario Internacional 20 Aniversario de la Red Iberoamericana de Investigadores sobre Globalización y Territorio RII (pp. 1-8). México: UANL, FARQ

Miralles, C. G., \& Cebollada, A. (2003). Movilidad y transporte: opciones políticas para la ciudad. España: Fundación de alternativas.

Miralles, C. G., \& Marquet, O. S. (2014). La proximidad en Barcelona: un análisis desde los tiempos de desplazamiento cotidianos. Revista Ciudades, 1(17). 99-120. Recuperado el 12 de enero de 2016, de https://dialnet.unirioja.es/ servlet/articulo? codigo $=4733392$

Mundó, J. T. (2002). El transporte colectivo urbano: aplicación del enfoque de sistemas para un mejor servicio. Fermentum, 12(34), 285-302. Recuperado el 19 de agosto de 2016, de http://www.redalyc.org/articulo.oa?id=70511239005

Romero, L. A. (2014, 07 de octubre). Análisis urbano del fraccionamiento hacienda real, en Tonalá, Jalisco. Blog ESARQ. Recuperado el 5 de diciembre de 2016, de https:// blog.esarq.edu.mx/2014/10/07/analisis-urbano-delfraccionamiento-hacienda-real-en-tonala-jalisco/

Sánchez, L. B. (2015). Porque no tenemos nada, queremos hacerlo todo. Diez años de movilidad en Guadalajara 
metropolitana. Revista Territorio sin Centro ni Periferia, 1. Recuperado el 9 de marzo de 2016, de http://www. revistaterritorio.mx/porque-no-tenemos-nada-queremoshacerlo-todo.html

Santos L. G. \& De las Rivas J. L. S. (2008). Ciudades con atributos: conectividad, accesibilidad y movilidad. Revista Ciudades, 11, 13-32. Recuperado el 10 de mayo de 2016, de http://www3.uva.es/iuu/REVISTA/Ciudades\%2011/ Ciudades\%2011\%20013032\%20SAN TOS\% 20y\%20 DE\%20LAS\%20RIVAS.pdf

Secretaría de Comunicaciones y Transportes - SCT. (2010). Información general. México: SCT. Recuperado el 10 de Junio de 2010, de http://www.sct.gob.mx/informaciongeneral/

Secretaría de Desarrollo Social - Sedesol. (2001). Programa nacional de desarrollo urbano y ordenación del territorio 2001-2006. Recuperado el 12 de septiembre de 2016, de www.sedesol.gob.mx

Silva, C. R., \& Burgos, C. D. (2011). Tiempo mínimoconocimiento suficiente: la cuasi etnografía sociotécnica en psicología social. Psicoperspectivas. 2(11), 87-108. Recuperado el 18 de enero de 2016, de http://www. psicoperspectivas.cl/index.php /psicoperspectivas/article/ viewFile/146/155

Smart Growth America. (2015). The best complete streets policies of 2014. Recuperado el 14 de mayo de 2016, de: http://www.smartgrowthamerica.org/documents/bestcomplete-streets-policies-of-2014.pdf

Social Exclusion Unit - SEU. (2003). Making the connections: final report on transport and social exclusion. London: Office of the Deputy Prime Minister.

United Nations Conference - ONU. (2016). Nueva Agenda Urbana: declaración de quito sobre ciudades y asentamientos humanos sostenibles para todos. Recuperado el 21 de diciembre de 2016, Recuperado de https://www2.habitat3. org/bitcache/907f3c56d3ad27a3daeeb677c660545a00c 69d6b?vid=591158\&disposition=inline\&op=view

Von Bertalanffy, L. (1976). Teoría general de los sistemas. México: Fondo de Cultura Económica.

Recibido: Jul. 13, 2017

Aprobado: Dic. 09, 2017 